Pacific Journal of Mathematic 


\title{
ON THE NUMBER OF SOLUTIONS OF $u^{k}+D \equiv w^{2}(\bmod p)$
}

\author{
Emma Lehmer
}

Introduction. The number $N_{k}(D)$ of solutions $(u, w)$ of the congruence

$$
u^{k}+D \equiv w^{2}(\bmod p)
$$

can be expressed in terms of the Gaussian cyclotomic numbers $(i, j)$ of order $\operatorname{LCM}(k, 2)$ as has been done by Vandiver [7], or in terms of the character sums introduced by Jacobsthal [4] and studied in special cases by von Schrutka [6], Chowla [1], and Whiteman [8]. In the special cases $k=3,4,5,6$, and 8, the answer can be expressed in terms of certain quadratic partitions of $p$, but unless $D$ is a $k$ th power residue there remained an ambiguity in sign, which we will be able to eliminate in some cases in the present paper. Theorems 2 and 4 were first conjectured from the numerical evidence provided by the SWAC and later proved by the use of cyclotomy. They improve Jacobsthal's results for all $p$ for which 2 is not a quartic residue. Similarly Theorem 6 improves von Schrutka's and Chowla's results for those $p$ 's which do not have 2 for a cubic residue. Only in case $k=2$ and in the cases where $k$ is oddly even and $D$ is a $(k / 2)$ th but not a $k$ th power residue is $N_{k}(D)$ a function of $p$ alone and is in fact $p-1$. This result appears in Theorem 1. In case $k=4$, Vandiver [7a] gives an unambiguous solution, which requires the determination of a primitive root.

1. Character sums. It is clear that the number of solutions $N_{k}(D)$ of (1) can be written

$$
N_{k}(D)=\sum_{u=0}^{p-1}\left[1+\left(\frac{u^{k}+D}{p}\right)\right]=p+\sum_{u=0}^{p-1}\left(\frac{u^{k}+D}{p}\right),
$$

or

$$
N_{k}(D)=p+\left(\frac{D}{p}\right)+\psi_{k}(D)
$$

Received July 10, 1953.

Pacific J. Math. 5 (1955), $103-118$ 
where the function

$$
\psi_{k}(D)=\sum_{u=1}^{p-1}\left(\frac{u^{k}+D}{p}\right)
$$

is connected with the Jacobsthal sum

$$
\phi_{k}(D)=\sum_{u=1}^{p-1}\left(\frac{u}{p}\right)\left(\frac{u^{k}+D}{p}\right)
$$

by the relations

$$
\psi_{k}(D)=\left(\frac{D}{p}\right) \phi_{k}(\bar{D}), k \text { odd and } D \bar{D} \equiv 1(\bmod p)
$$

and

$$
\psi_{2 k}(D)=\psi_{k}(D)+\phi_{k}(D)
$$

Other pertinent relations are

$$
\left\{\begin{array}{l}
\phi_{k}\left(m^{k} D\right)=\left(\frac{m}{p}\right)^{k+1} \phi_{k}(D) \\
\psi_{k}\left(m^{k} D\right)=\left(\frac{m}{p}\right)^{k} \psi_{k}(D)
\end{array}\right.
$$

and

(8)

$$
\left\{\begin{array}{l}
\phi_{k}(\bar{D})=-\left(\frac{D}{p}\right) \phi_{k}(D) \\
\psi_{k}(\bar{D})=\left(\frac{D}{p}\right) \psi_{k}(D) .
\end{array}\right.
$$

Also, for $k$ odd and $\rho$ a primitive root,

$$
\sum_{\nu=0}^{k-1} \phi_{k}\left(\rho^{\nu}\right)=-k
$$

These relations are either well known or are paraphrases of known relations 
and are all easily derivable from the definitions. If $k$ is odd, it follows from (5) and (6) that

$$
\psi_{2 k}(D)=\phi_{k}(D)+\left(\frac{D}{p}\right) \phi_{k}(\bar{D})
$$

If $D$ is a $k$ th power residue, then so is $\bar{D}$ and hence by (7) for $k$ odd $\phi_{k}(D)=$ $\phi_{k}(\bar{D})=\phi_{k}(1)$, and we have

$$
\psi_{2 k}(D)=\phi_{k}(D)\left[1+\left(\frac{D}{p}\right)\right]= \begin{cases}2 \phi_{k}(D) & \text { if }\left(\frac{D}{p}\right)=+1 \\ 0 & \text { if }\left(\frac{D}{p}\right)=-1\end{cases}
$$

Hence from (2) we obtain:

THEOREM l. If $k$ is odd and if $D=m^{k}$, where $m$ is a nonresidue of $p=2 k h+1$, then the number $N_{2 k}\left(m^{k}\right)$ of solutions $(u, w)$ of

$$
u^{2 k}+m^{k} \equiv w^{2}(\bmod p)
$$

is exactly $p-1$.

Since $\phi_{1}(D)=-1$, it follows from $(11)$ that $\psi_{2}(D)=-2$, if $D$ is a residue, and zero otherwise. Hence by $(2), N_{2}(D)=p-1$ for all $D$. This is a well known result in quadratic congruences. We will next discuss the case $k=4$, which is connected with Jacobsthal's theorem.

Jacobsthal [4] proved that if $D$ is a residue and if $p=x^{2}+4 y^{2}$, then

$$
\phi_{2}(D)=-2 x\left(\frac{\sqrt{D}}{p}\right), \quad x \equiv 1(\bmod 4) ;
$$

but if $D$ is a nonresidue then he was able to prove only that

$$
\phi_{2}(D)= \pm 4 y
$$

Hence for $D$ a residue, it follows from the fact that $\psi_{2}(D)=-2$, using $(6)$ and (2), that

$$
N_{4}(D)=p-1-2 x\left(\frac{\sqrt{D}}{p}\right), \quad x \equiv 1(\bmod 4) .
$$


However, the corresponding result for $D$ nonresidue would read

$$
N_{4}(D)=p-1 \pm 4 y \text {. }
$$

In order to eliminate this ambiguity in sign at least for some cases we now turn to the cyclotomic approach.

2. Cyclotomy. If we define as usual the cyclotomic number $(i, j)_{k}$ as the number of solutions $(\nu, \mu)$ of the congruence

$$
g^{k \nu+i}+1 \equiv g^{k \mu+j} \quad(\bmod p)
$$

then if $D$ belongs to class $s$ with respect to some primitive root $g$ (that is, if $\left.\operatorname{ind}_{g} D \equiv s(\bmod k)\right)$, we can write the number of nonzero solutions of $(1)$ for $k$ even as follows:

$$
N_{k}^{*}(D)=2 k \sum_{\nu=1}^{k / 2}(k-s, 2 \nu-s)_{k} .
$$

We now assume that 2 is a nonresidue and choose $g$ so that 2 belongs to the first class, or $s=1$; then

$$
N_{4}(2)=N_{4}^{*}(2)=8\left[(3,1)_{4}+(3,3)_{4}\right] \text {. }
$$

These cyclotomic constants have been calculated by Causs [3] in terms of $x$ and $y$ in the quadratic partition $p=x^{2}+4 y^{2}$ and are for $p=8 n+5$

$$
16(3,3)_{4}=p-2 x-3, \quad 16(3,1)_{4}=p+2 x-8 y+1 .
$$

Substituting this into (18) we obtain

$$
N_{4}(2)=p-1-4 y, \quad\left(\frac{2}{p}\right)=-1
$$

To determine the sign of $y$ we recall a lemma of our previous paper [5] which states that $(0, s)$ is odd or even according as 2 belongs to class $s$ or not. Hence in our case $(0,0)$ is even, while $(0,1)$ is odd. These numbers have been given by Gauss as follows,

$$
16(0,0)_{4}=p+2 x-7, \quad 16(0,1)_{4}=p+2 x+8 y+1 .
$$

\section{Hence}




$$
p+2 x-7 \equiv 0(\bmod 32) \text { and } p+2 x+8 y+1 \equiv 16(\bmod 32) \text {. }
$$

Subtracting the first congruence from the second we have, dividing by 8 ,

$$
y \equiv 1(\bmod 4)
$$

This makes (20) unambiguous, and returning to (2) we find by (6), since $\psi_{2}(2)=0$, that for $(2 / p)=-1$

$$
\psi_{4}(2)=\phi_{2}(2)=-4 y, \quad y \equiv 1(\bmod 4)
$$

Hence by ( 7)

$$
\phi_{2}\left(2 m^{2}\right)=-4 y\left(\frac{m}{p}\right), \quad\left(\frac{2}{p}\right)=-1
$$

This gives a slight strengthening of Jacobsthal's theorem, namely:

THEOREM 2. If 2 is a nonresidue of $p=x^{2}+4 y^{2}$, where $x \equiv y \equiv 1(\bmod 4)$, then

$$
\phi_{2}(D)=\left\{\begin{array}{l}
-2 x\left(\frac{m}{p}\right), \text { if } D \equiv m^{2}(\bmod p) \\
-4 y\left(\frac{m}{p}\right), \text { if } D \equiv 2 m^{2}(\bmod p) .
\end{array}\right.
$$

Hence by (2) we have:

THEOREM 3. If 2 is a nonresidue of $p=x^{2}+4 y^{2}, x \equiv y \equiv 1(\bmod 4)$ then the number of solutions of $u^{4}+D \equiv w^{2}(\bmod p)$ is given by

$$
N_{4}(D)= \begin{cases}p-1-2 x\left(\frac{m}{p}\right), & \text { if } D \equiv m^{2}(\bmod p) \\ p-1-4 y\left(\frac{m}{p}\right), & \text { if } D \equiv 2 m^{2}(\bmod p) .\end{cases}
$$

We now suppose that 2 is a quadratic residue but a quartic nonresidue, hence we may choose $g$ such that $\sqrt{2}$ belongs to class 1 and calculate $N(\sqrt{2})$ by (18). The cyclotomic constants of order 4 for $p=8 n+1$ are

$$
16(3,1)_{4}=p-2 x+1, \quad 16(3,3)_{4}=p+2 x+8 y-3
$$


Hence by (18)

$$
N_{4}(\sqrt{2})=p-1+4 y
$$

but in this case $y$ turns out to be even, so that it is not sufficient to determine $y$ modulo 4 and it is necessary to introduce the cyclotomic numbers of order 8 to determine the sign of $y$. It also becomes necessary to distinguish the cases $p=16 n+1$ and $16 n+9$.

$$
\text { Case 1. } p=16 n+1=x^{2}+4 y^{2}=a^{2}+2 b^{2}, x \equiv a \equiv 1(\bmod 4) .
$$

Since $\sqrt{2}$ belongs to class 1,2 belongs to class 2 and by our lemma $(0,0)_{8}$ is even, while $(0,2)_{8}$ is odd. Dickson [2] gives

$$
64(0,0)_{8}=p-23+6 x \text {. }
$$

Since $(0,0)_{8}$ is even, we have

$$
6 x \equiv-p+23(\bmod 128)
$$

In order to complete our discussion it was necessary to calculate $(0,2)_{8}$ and $(1,2)_{8}$ by solving 15 linear equations involving the constants $(i, j)_{8}$ given by Dickson, which we list in the Appendix. We obtained

$$
64(0,2)_{8}=p-7-2 x-16 y-8 a, 64(1,2)_{8}=p+1-6 x+4 a .
$$

Substituting $p-23$ for $-6 x$ from $(28)$ into $64(1,2)_{8}$ we obtain

$$
2 a \equiv 11-p(\bmod 32) \text {. }
$$

Since $(0,2)_{8}$ is odd we have, multiplying $(29)$ by 3 ,

$$
\begin{aligned}
3 p-21-6 x-48 y-24 a \equiv 3 p-21+(p-23)-48 y- & 12(11-p) \\
& \equiv 64(\bmod 128) ;
\end{aligned}
$$

or, dividing out a 16 and solving for $y$, we get

$$
y \equiv 3(p+1) \equiv-2(\bmod 8) \text {. }
$$

Case 2. $p=16 n+9$. In this case Dickson gives

$$
64(0,4)_{8}=p+1+6 x+24 a,
$$


while we have calculated [ see Appendix]

$$
\begin{aligned}
& 64(0,2)_{8}=p+1-2 x+16 y, \\
& 64(2,0)_{8}=p-7+6 x, \\
& 64(1,2)_{8}=p+1+2 x-4 a .
\end{aligned}
$$

From (35)

$$
6 x \equiv 7-p(\bmod 64) .
$$

Substituting this into (36) we find

$$
12 a \equiv 2 p+10(\bmod 64) .
$$

Since $(0,4)_{8}$ is even we obtain, using $(38)$,

$$
p+1+6 x+24 a \equiv p+1+6 x+4 p+20 \equiv 0(\bmod 128) .
$$

This gives an improvement of (37), namely,

$$
6 x \equiv-(5 p+21)(\bmod 128) .
$$

Finally substituting all this into $(0,2)_{8}$ which is odd, we have, after multiplying (34) by 3 ,

$$
3 p+3-6 x+48 y \equiv 3 p+3+5 p+21+48 y \equiv 8 p+24+48 y \equiv 64(\bmod 128),
$$

or dividing out an 8 and noting that $p \equiv 9(\bmod 16)$ we obtain

$$
y \equiv+2(\bmod 8) \text {. }
$$

Hence the sign of $y$ in $(26)$ is now determined as follows if $(\sqrt{2} / p)=-1$ :

$$
N_{4}(\sqrt{2})=p-1+4 y, \text { where } y / 2 \equiv-(-1)^{(p-1) / 8}(\bmod 4) .
$$

From this we have as before by $(2)$ and $(6)$ for $(\sqrt{2} / p)=-1$ :

$$
\psi_{4}(\sqrt{2})=\phi_{2}(\sqrt{2})=-4 y \text {, where } y / 2 \equiv(-1)^{(p-1) / 8}(\bmod 4) \text {, }
$$

and we can write a slight improvement of Jacobsthal's theorem in the case in which 2 is a quadratic but not a quartic residue of $p$ : 
THEOREM 4. If 2 is a quadratic residue, but a quartic nonresidue of $p=$ $x^{2}+4 y^{2}=8 n+1$, then

$$
\phi_{2}(D)=\left\{\begin{array}{l}
-2 x\left(\frac{m}{p}\right) \text { if } D \equiv m^{2}(\bmod p) \\
-4 y\left(\frac{m}{p}\right) \text { if } D \equiv \sqrt{2 m^{2}}(\bmod p),
\end{array}\right.
$$

where $x \equiv 1(\bmod 4)$ and $y / 2 \equiv(-1)^{n}(\bmod 4)$.

THEOREM 5. If 2 is a quadratic residue, but a quartic nonresidue of $p=$ $x^{2}+4 y^{2}=8 n+1$, then the number of solutions $(u, w)$ of $u^{4}+D \equiv w^{2}(\bmod p)$ is given by

$$
N_{4}(D)=\left\{\begin{array}{l}
p-1-2 x\left(\frac{m}{p}\right) \text { if } D \equiv m^{2}(\bmod p) \\
p-1-4 y\left(\frac{m}{p}\right) \text { if } D \equiv \sqrt{2 m}^{2}(\bmod p),
\end{array}\right.
$$

where $x \equiv 1(\bmod 4)$ and $y / 2 \equiv(-1)^{n}(\bmod 4)$.

In order to obtain an improvement on Jacobsthal's theorem in the case in which 2 is a quartic residue, or to improve the results for $\phi_{4}$ and $\psi_{4}$ in order to obtain $N_{8}$, it appears necessary to examine the cyclotomic constants of order 16, or to go through a determination of a specified primitive root as in Vandiver $[\mathbf{7 a}]$. The known results for $\phi_{4}$ and $\psi_{4}$ are as follows:

$$
\phi_{4}(D)= \begin{cases}-4 a\left(\frac{m}{p}\right) & \text { if } D \equiv m^{4}(\bmod p) \\ 0 & \text { if } D \equiv m^{2} \not \equiv m_{1}^{4}(\bmod p) \\ \pm 4 b & \text { otherwise }\end{cases}
$$

and

$$
\psi_{4}(D)= \begin{cases}-2 x\left(\frac{m}{p}\right)-2 & \text { if } D \equiv m^{2}(\bmod p) \\ \pm 4 y & \text { otherwise. }\end{cases}
$$

It follows from this that 


$$
N_{8}(D)= \begin{cases}p-1-2 x-4 a\left(\frac{m}{p}\right) & \text { if } D \equiv m^{4}(\bmod p) \\ p-1+2 x\left(\frac{m}{p}\right) & \text { if } D \equiv m^{2} \not \equiv m_{1}^{4}(\bmod p) \\ p-1 \pm 4 b \pm 4 y & \text { otherwise. }\end{cases}
$$

3. Case $k=3$. The known results for the case $k=3$ can be stated as follows:

$$
\phi_{3}(D)= \begin{cases}-2 A-1 & \text { if } D \text { is a cubic residue } \\ A \pm 3 B-1 & \text { if } D \text { is a cubic nonresidue }\end{cases}
$$

where $p=A^{2}+3 B^{2}=6 n+1, A \equiv 1(\bmod 3)$.

This can be obtained either by summing the appropriate cyclotomic constants of order 6, or by using the results of Schrutka or Chowla, as was done in Whiteman [8]. From this it follows by (2) and (5) that

$$
N_{3}(D)= \begin{cases}p-\left(\frac{D}{p}\right) 2 A & \text { if } D \text { is a cubic residue } \\ p+\left(\frac{D}{p}\right)(A \pm 3 B) & \text { if } D \text { is a cubic nonresidue. }\end{cases}
$$

We are again faced with an ambiguity in sign in case $D$ is a cubic nonresidue, which can be resolved in case 2 is a cubic nonresidue. For in this case by (9)

$$
\phi_{3}(1)+\phi_{3}(2)+\phi_{3}(4)=-3
$$

By (44), $\phi_{3}(1)=-2 A-1$, while Chowla proved that $\phi_{3}(4)=L-1$, where $4 p=L^{2}+27 M^{2}, L \equiv 1(\bmod 3)$. Hence by $(46)$

$$
\phi_{3}(2)=2 A-L-1 \quad(2 \text { a cubic nonresidue })
$$

Hence by (7) we can write a slight generalization of Chowla's or Schrutka's theorem:

THEOREM 6. If 2 is a cubic nonresidue of $p=A^{2}+3 B^{2}$, and if $4 p=L^{2}+$ $27 M^{2}, A \equiv L \equiv 1(\bmod 3)$, then 


$$
\phi_{3}(D)= \begin{cases}-(2 A+1) & \text { if } D \equiv m^{3}(\bmod p) \\ 2 A-L-1 & \text { if } D \equiv 2 m^{3}(\bmod p) \\ L-1 & \text { if } D \equiv 4 m^{3}(\bmod p)\end{cases}
$$

Using (5) and (2) we obtain the corresponding theorem for $N_{3}(D)$ :

THEOREM 7. If 2 is a cubic nonresidue of $p=A^{2}+3 B^{2}$, and if $4 p=L^{2}+$ $27 M^{2}, A \equiv L \equiv 1(\bmod 3)$, then

$$
N_{3}(D)= \begin{cases}p-\left(\frac{D}{p}\right) 2 A & \text { if } D \equiv m^{3}(\bmod p) \\ p+\left(\frac{D}{p}\right) L & \text { if } D \equiv 2 m^{3}(\bmod p) \\ p+\left(\frac{D}{p}\right)(2 A-L) & \text { if } D \equiv 4 m^{3}(\bmod p) .\end{cases}
$$

For $k=6$, it follows from $(10)$ by substituting the values for $\phi_{3}(D)$ from (44) (remembering that $D$ and $\bar{D}$ are either both cubic residues, or both nonresidues), that:

$$
\psi_{6}(D)=\left\{\begin{array}{l}
-(2 A+1)\left[1+\left(\frac{D}{p}\right)\right] \text { if } D \text { is a cubic residue } \\
(A-1)\left[1+\left(\frac{D}{p}\right)\right] \pm 3 B\left[1-\left(\frac{D}{p}\right)\right] \text { otherwise. }
\end{array}\right.
$$

Substituting this into (2) we have

$$
N_{6}(D)=\left\{\begin{array}{l}
p-2 A\left[1+\left(\frac{D}{p}\right)\right]-1 \text { if } D \text { is a cubic residue } \\
p+A\left[1+\left(\frac{D}{p}\right)\right] \pm 3 B\left[1-\left(\frac{D}{p}\right)\right]-1 \text { otherwise }
\end{array}\right.
$$

In case 2 is a cubic nonresidue, however, we can substitute more exact values for $\phi_{3}(D)$ from Theorem 6 into (10) to obtain:

THEOREM 7. If 2 is a cubic nonresidue of $p=A^{2}+3 B^{2}$ and if $4 p=L^{2}+$ $27 M^{2} . A \equiv L \equiv 1(\bmod 3)$, then 


$$
\psi_{6}(D)= \begin{cases}-(2 A+1)\left[1+\left(\frac{D}{p}\right)\right] & \text { if } D \equiv m^{3}(\bmod p) \\ 2 A+L\left[\left(\frac{D}{p}\right)-1\right]-\left[1+\left(\frac{D}{p}\right)\right] & \text { if } D \equiv 2 m^{3}(\bmod p) \\ \left(\frac{D}{p}\right) 2 A-L\left[\left(\frac{D}{p}\right)-1\right]-\left[1+\left(\frac{D}{p}\right)\right] & \text { if } D \equiv 4 m^{3}(\bmod p) .\end{cases}
$$

Substituting these values intc (2) we obtain:

THEOREM 8. If 2 is a cubic nonresidue of $p=A^{2}+3 B^{2}$ and if $4 p=L^{2}+$ $27 M^{2}, A \equiv L \equiv 1(\bmod 3)$, then the number of solutions of $u^{6}+D \equiv v^{2}(\bmod p)$ is given by

$$
N_{6}(D)= \begin{cases}p-1-2 A\left[1+\left(\frac{D}{p}\right)\right] & \text { if } D \equiv m^{3}(\bmod p) \\ p-1+2 A+L\left[\left(\frac{D}{p}\right)-1\right] & \text { if } D \equiv 2 m^{3}(\bmod p) \\ p-1+\left(\frac{D}{p}\right) 2 A-L\left[\left(\frac{D}{p}\right)-1\right] & \text { if } D \equiv 4 m^{3}(\bmod p) .\end{cases}
$$

4. Congruences in three variables. In conclusion we can apply our results to the number of solutions of congruences in three variables. We have:

THEOREM 9. The number $N_{k, k}(D)$ of solutions $(u, v, w)$ of

$$
u^{k}+D v^{k} \equiv w^{2}(\bmod p)
$$

is

$$
N_{k, k}(D)= \begin{cases}p^{2} & \text { if } k \text { is odd } \\ p^{2}+(p-1)\left[1+\left(\frac{D}{p}\right)+\psi_{k}(D)\right] & \text { if } k \text { is even } .\end{cases}
$$

Proof. Replacing $D$ by $D \nu^{k}$ in (2) and summing over $\nu=1,2, \cdots, p-1$, we obtain

$$
\sum_{\nu=1}^{p-1} N_{k}\left(D \nu^{k}\right)=p(p-1)+\left(\frac{D}{p}\right) \sum_{\nu=1}^{p-1}\left(\frac{\nu}{p}\right)^{k}+\sum_{\nu=1}^{p-1} \psi_{k}\left(\nu^{k} D\right) .
$$


By (7) this becomes

$$
\sum_{\nu=1}^{p-1} N_{k}\left(D \nu^{k}\right)=p(p-1)+\left(\frac{D}{p}\right) \sum_{\nu=1}^{p-1}\left(\frac{\nu}{p}\right)^{k}+\psi_{k}(D) \sum_{\nu=1}^{p-1}\left(\frac{\nu}{p}\right)^{k}
$$

But

$$
\sum_{\nu=1}^{p-1}\left(\frac{\nu}{p}\right)^{k}= \begin{cases}0 & k \text { odd } \\ p-1 & k \text { even }\end{cases}
$$

while the number of solutions with $\nu=0$ is $p$ for $k$ odd and $2 p-1$ for $k$ even. Hence

$$
N_{k, k}(D)=\left\{\begin{array}{lr}
p(p-1)+p=p^{2} & \text { for } k \text { odd } \\
p(p-1)+(p-1)\left[\left(\frac{D}{p}\right)+\psi_{k}(D)\right]+2 p-1, & k \text { even } .
\end{array}\right.
$$

Hence the theorem.

Using the expressions derived for special values of $k$ earlier we can write down the following special cases:

$$
N_{2,2}(D)=p^{2}
$$

By (14),

$$
N_{4,4}(D)=p^{2}-2 x\left(\frac{\sqrt{D}}{p}\right)(p-1) \quad \text { if }\left(\frac{D}{p}\right)=+1, x \equiv 1(\bmod 4) .
$$

By (24),

$$
N_{4,4}\left(2 m^{2}\right)=p^{2}-4 y(p-1) \quad \text { if }\left(\frac{2}{p}\right)=-1 \text { and } y \equiv 1(\bmod 4)
$$

By (42),

$$
N_{4,4}\left(\sqrt{2 m^{2}}\right)=p^{2}-4 y(p-1) \quad \text { if } \quad \frac{\sqrt{2}}{p}=-1 \text { and } y / 2 \equiv(-1)^{(p-1) / 8}(\bmod 4) .
$$

By (48), 


$$
N_{6,6}\left(m^{3}\right)=p^{2}-2 A\left[1+\left(\frac{m}{p}\right)\right](p-1) .
$$

By Theorem 7,

$$
\left.\begin{array}{l}
N_{6,6}\left(2 m^{3}\right)=p^{2}+\left\{2 A+L\left[\left(\frac{m}{p}\right)-1\right]\right\}(p-1) \\
N_{6,6}\left(4 m^{3}\right)=p^{2}+\left\{\left(\frac{m}{p}\right) 2 A-L\left[\left(\frac{m}{p}\right)-1\right]\right\}(p-1)
\end{array}\right\} \text { if } 2 \text { is a cubic nonresidue. }
$$

By (43),

$$
N_{8,8}\left(m^{4}\right)=p^{2}-\left[2 x+4 a\left(\frac{m}{p}\right)\right](p-1) .
$$

We note that $N_{6,6}\left(m^{3}\right)=p^{2}$ if $m$ is a nonresidue. It can be readily seen that this is a special case of a general theorem, namely:

THEOREM 10. If $k$ is oddly even and $D$ is a $k / 2$ th power residue, but not a $k$ th power residue, then

$$
N_{k, k}(D)=p^{2}
$$

This follows from Theorem 9 and the fact that the corresponding $\psi_{k}(D)$ is zero in this case by (11).

We hope to take up the cases $k=5$ and $k=10$ in a future paper.

\section{APPENDIX: Cyclotomic constants of order 8.}

The 64 constants $(i, j)_{8}$ have at most 15 different values for a given $p$. These values are expressible in terms of $p, x, y, a$ and $b$ in

$$
p=x^{2}+4 y^{2}=a^{2}+2 b^{2}, \quad(x \equiv a \equiv 1(\bmod 4)) .
$$

There are two cases.

Case I. $p=16 n+1$. 
Table of $(i, j)_{8}$

\begin{tabular}{c|cccccccc}
$j$ & 0 & 1 & 2 & 3 & 4 & 5 & 6 & 7 \\
\hline 0 & $(0,0)$ & $(0,1)$ & $(0,2)$ & $(0,3)$ & $(0,4)$ & $(0,5)$ & $(0,6)$ & $(0,7)$ \\
1 & $(0,1)$ & $(0,7)$ & $(1,2)$ & $(1,3)$ & $(1,4)$ & $(1,5)$ & $(1,6)$ & $(1,2)$ \\
2 & $(0,2)$ & $(1,2)$ & $(0,6)$ & $(1,6)$ & $(2,4)$ & $(2,5)$ & $(2,4)$ & $(1,3)$ \\
3 & $(0,3)$ & $(1,3)$ & $(1,6)$ & $(0,5)$ & $(1,5)$ & $(2,5)$ & $(2,5)$ & $(1,4)$ \\
4 & $(0,4)$ & $(1,4)$ & $(2,4)$ & $(1,5)$ & $(0,4)$ & $(1,4)$ & $(2,4)$ & $(1,5)$ \\
5 & $(0,5)$ & $(1,5)$ & $(2,5)$ & $(2,5)$ & $(1,4)$ & $(0,3)$ & $(1,3)$ & $(1,6)$ \\
6 & $(0,6)$ & $(1,6)$ & $(2,4)$ & $(2,5)$ & $(2,4)$ & $(1,3)$ & $(0,2)$ & $(1,2)$ \\
7 & $(0,7)$ & $(1,2)$ & $(1,3)$ & $(1,4)$ & $(1,5)$ & $(1,6)$ & $(1,2)$ & $(0,1)$
\end{tabular}

These 15 fundamental constants $(0,0), \ldots,(2,5)$ are given by the relations contained in the following table.

If 2 is a quartic residue If 2 is not a quartic residue

\begin{tabular}{l|ll}
$64(0,0)$ & $p-23-18 x-24 a$ & $p-23+6 x$ \\
$64(0,1)$ & $p-7+2 x+4 a+16 y+16 b$ & $p-7+2 x+4 a$ \\
$64(0,2)$ & $p-7+6 x+16 y$ & $p-7-2 x-8 a-16 y$ \\
$64(0,3)$ & $p-7+2 x+4 a-16 y+16 b$ & $p-7+2 x+4 a$ \\
$64(0,4)$ & $p-7-2 x+8 a$ & $p-7-10 x$ \\
$64(0,5)$ & $p-7+2 x+4 a+16 y-16 b$ & $p-7+2 x+4 a$ \\
$64(0,6)$ & $p-7+6 x-16 y$ & $p-7-2 x-8 a+16 y$ \\
$64(0,7)$ & $p-7+2 x+4 a-16 y-16 b$ & $p-7+2 x+4 a$ \\
$64(1,2)$ & $p+1+2 x-4 a$ & $p+1-6 x+4 a$ \\
$64(1,3)$ & $p+1-6 x+4 a$ & $p+1+2 x-4 a-16 b$ \\
$64(1,4)$ & $p+1+2 x-4 a$ & $p+1+2 x-4 a+16 y$ \\
$64(1,5)$ & $p+1+2 x-4 a$ & $p+1+2 x-4 a-16 y$ \\
$64(1,6)$ & $p+1-6 x+4 a$ & $p+1+2 x-4 a+16 b$ \\
$64(2,4)$ & $p+1-2 x$ & $p+1+6 x+8 a$ \\
$64(2,5)$ & $p+1+2 x-4 a$ & $p+1-6 x+4 a$
\end{tabular}


Case II. $p=16 n+9$.

Table of $(i, j)_{8}$

\begin{tabular}{c|cccccccc}
$j$ & 0 & 1 & 2 & 3 & 4 & 5 & 6 & 7 \\
\hline 0. & $(0,0)$ & $(0,1)$ & $(0,2)$ & $(0,3)$ & $(0,4)$ & $(0,5)$ & $(0,6)$ & $(0,7)$ \\
1 & $(1,0)$ & $(1,1)$ & $(1,2)$ & $(1,3)$ & $(0,5)$ & $(1,3)$ & $(0,3)$ & $(1,7)$ \\
2 & $(2,0)$ & $(2,1)$ & $(2,0)$ & $(1,7)$ & $(0,6)$ & $(1,3)$ & $(0,2)$ & $(1,2)$ \\
3 & $(1,1)$ & $(2,1)$ & $(2,1)$ & $(1,0)$ & $(0,7)$ & $(1,7)$ & $(1,2)$ & $(0,1)$ \\
4 & $(0,0)$ & $(1,0)$ & $(2,0)$ & $(1,1)$ & $(0,0)$ & $(1,0)$ & $(2,0)$ & $(1,1)$ \\
5 & $(1,0)$ & $(0,7)$ & $(1,7)$ & $(1,2)$ & $(0,1)$ & $(1,1)$ & $(2,1)$ & $(2,1)$ \\
6 & $(2,0)$ & $(1,7)$ & $(0,6)$ & $(1,3)$ & $(0,2)$ & $(1,2)$ & $(2,0)$ & $(2,1)$ \\
7 & $(1,1)$ & $(1,2)$ & $(1,3)$ & $(0,5)$ & $(0,3)$ & $(1,6)$ & $(1,3)$ & $(1,0)$
\end{tabular}

where

If 2 is a quartic residue If 2 is not a quartic residue

\begin{tabular}{l|ll}
$64(0,0)$ & $p-15-2 x$ & $p-15-10 x-8 a$ \\
$64(0,1)$ & $p+1+2 x-4 a+16 y$ & $p+1+2 x-4 a-16 b$ \\
$64(0,2)$ & $p+1+6 x+8 a-16 y$ & $p+1-2 x+16 y$ \\
$64(0,3)$ & $p+1+2 x-4 a-16 y$ & $p+1+2 x-4 a-16 b$ \\
$64(0,4)$ & $p+1-18 x$ & $p+1+6 x+24 a$ \\
$64(0,5)$ & $p+1+2 x-4 a+16 y$ & $p+1+2 x-4 a+16 b$ \\
$64(0,6)$ & $p+1+6 x+8 a+16 y$ & $p+1-2 x-16 y$ \\
$64(0,7)$ & $p+1+2 x-4 a-16 y$ & $p+1+2 x-4 a+16 b$ \\
$64(1,0)$ & $p-7+2 x+4 a$ & $p-7+2 x+4 a+16 y$ \\
$64(1,1)$ & $p-7+2 x+4 a$ & $p-7+2 x+4 a-16 y$ \\
$64(1,2)$ & $p+1-6 x+4 a+16 b$ & $p+1+2 x-4 a$ \\
$64(1,3)$ & $p+1+2 x-4 a$ & $p+1-6 x+4 a$ \\
$64(1,7)$ & $p+1-6 x+4 a-16 b$ & $p+1+2 x-4 a$ \\
$64(2,0)$ & $p-7-2 x-8 a$ & $p-7+6 x$ \\
$64(2,1)$ & $p+1+2 x-4 a$ & $p+1-6 x+4 a$
\end{tabular}




\section{REFERENCES}

1. S. Chowla, The last entry in Gauss' diary, Proc. Nat. Acad. Sci. U.S.A. 35 (1949), $244-246$.

2. L. E. Dickson, Cyclotomy, higher congruences and Waring's problem, Amer. J. Math. 57 (1935), $391-424$.

3. C. F. Gauss, Theoria residuorum biquadraticorum, Werke, $2,90$.

4. E. Jacobsthal, Anwendungen einer Formel aus der Theorie der quadratischen Reste, Dissertation (Berlin 1906).

5. Emma Lehmer, On residue difference sets, Canadian J. Math. 5 (1953), 425-432.

6. L. von Schrutka, Eine Beweis für die Zerlegbarkeit der Primzahlen von der Form $6 n+1$ in ein einfaches und ein dreifaches Quadrat, Jn. für die reine und angew. Math. 140 (1911), $252-265$.

7. H.S. Vandiver, On the number of solutions of some general types of equations in a finite field, Proc. Nat. Acad. Sci. U.S.A. 32 (1946), 47-52.

7a. - On the number of solutions of certain nonhomogeneous trinomial equations in a finite field, Proc. Nat. Acad. Sci. U.S.A. 31 (1945), 170-175.

8. A. L. Whiteman, Cyclotomy and Jacobsthal's sums, Amer. J. Math., 64 (1952), 89-99, and Theorems analogous to Jacobsthal's theorem, Duke Math. J. 16 (1949), $619-626$.

Berkeley, California 



\section{Pacific Journal of Mathematics}

\section{Vol. 5, No. $1 \quad$ September, 1955}

Frank Herbert Brownell, III, Flows and noncommuting projections on

Hilbert space ................................... 1

H. E. Chrestenson, A class of generalized Walsh functions ............. 17

Jean Bronfenbrenner Crockett and Herman Chernoff, Gradient methods of maximization ................................... 33

Nathan Jacob Fine, On groups of orthonormal functions. I .......... 51

Nathan Jacob Fine, On groups of orthonormal functions. II ............ 61

Frederick William Gehring, A note on a paper by L. C. Young .......... 67

Joachim Lambek and Leo Moser, On the distribution of Pythagorean

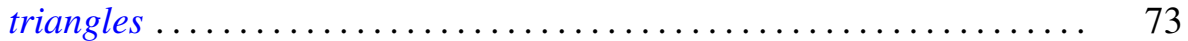

Roy Edwin Wild, On the number of primitive Pythagorean triangles with

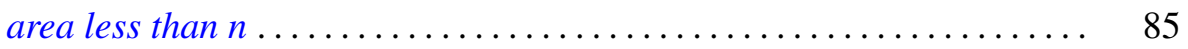

R. Sherman Lehman, Approximation of improper integrals by sums over multiples of irrational numbers ........................ 93

Emma Lehmer, On the number of solutions of $u^{k}+D \equiv w^{2}(\bmod p) \ldots 103$

Robert Delmer Stalley, A modified Schnirelmann density............... 119

Richard Allan Moore, The behavior of solutions of a linear differential equation of second order............................. 125

William M. Whyburn, A nonlinear boundary value problem for second order differential systems. 